\title{
ORIGINAL ARTICLE \\ Colorectal transport during defecation in subjects with supraconal spinal cord injury
}

\author{
MM Rasmussen ${ }^{1,2}, \mathrm{~K} \mathrm{Krogh}^{3}$, D Clemmensen ${ }^{2}, \mathrm{H}_{\text {Bluhme }}^{4}$, Y Rawashdeh ${ }^{5}$ and P Christensen ${ }^{1}$
}

Study design: Clinical study.

Objectives: To explore how supraconal spinal cord injury (SCI) affects colorectal emptying at defecation. Further, to relate findings to subject symptomatology expressed by bowel function scores and gastrointestinal transit time (GITT).

Setting: Aarhus University Hospital, Denmark.

Methods: Colorectal contents were marked by oral intake of ${ }^{111}$ In-coated resin pellets. Movement of stools at defecation was assessed by comparing scintigrams performed before and just after defecation. Results from 15 subjects with SCl ( 14 males, median age $=47$ years (range: 22-74 years), SCl level: C5-Th9) were compared with those from 16 healthy volunteers (12 males, median age = 31 years (range: 24-42 years)). Bowel symptoms were described from standard symptom scores, and GITT was assessed by radiopaque markers.

Results: Median emptying at defecation was 31\% of the rectosigmoid (range: $0 \%$ to complete emptying of the rectosigmoid and $49 \%$ of the descending colon) in subjects with $\mathrm{SCl}$ and $89 \%$ of the rectosigmoid (range: $53 \%$ to complete emptying of the rectosigmoid and the descending colon, and $3 \%$ of the transverse colon) in the control group $(P<0.01)$. Colorectal emptying at defecation was associated with the St Mark's fecal incontinence score $(P=0.02)$ but not with the Cleveland constipation score $(P=0.17)$, the neurogenic bowel dysfunction score $(P=0.12)$ or GITT $(P=0.99)$.

Conclusion: Supraconal SCl results in significantly reduced emptying of stools at defecation. This is independent of changes in GITT. Spinal Cord (2013) 51, 683-687; doi:10.1038/sc.2013.58; published online 18 June 2013

Keywords: neurogenic bowel dysfunction; supraconal spinal cord injury; colorectal scintigraphy; defecation; constipation

\section{INTRODUCTION}

Symptoms of neurogenic bowel dysfunction (NBD) are among of the most troublesome problems facing subjects with spinal cord injury (SCI). Difficult bowel emptying is a prominent symptom. ${ }^{1}$ The pathophysiological effect on bowel function depends on lesion level. Subjects with supraconal SCI generally have prolonged colonic transit, whereas those with conal and cauda equina lesions primarily have rectosigmoid impaction. ${ }^{2}$ Supraconal injuries result in a hypertone and hyperreactive rectosigmoid wall, reversely for conal and cauda equina injuries. ${ }^{3}$ Therefore, results obtained among subjects with conal and cauda equina lesions are not directly applicable to lesions at higher levels.

Quantification of intraluminal transport at defecation in supraconal SCI has never been performed. A direct measurement is possible when stools are marked with orally taken radioactive isotopes followed by colorectal scintigraphy before and after defecation.

The standard method for objective evaluation of colorectal or gastrointestinal transit time (GITT) is oral intake of radiopaque markers followed by one or more plain abdominal X-rays. ${ }^{4}$ This method is also followed for $\mathrm{SCI}, 2,5,6$ but there is no correlation between subject-reported symptoms of NBD and colorectal transit time or GITT assessed by radiopaque markers. ${ }^{5}$

Based on this, we hypothesized that subjects with supraconal SCI have reduced colorectal transport at defecation. Accordingly, we aimed at comparing colorectal transport and bowel emptying during defecation in subjects with supraconal SCI and healthy volunteers. Further, we aimed at investigating possible correlations between transport at defecation and symptoms of NBD and GITT.

\section{MATERIALS AND METHODS}

Subjects eligible for inclusion were identified via file review in our neurosurgical department (Figure 1). Inclusion criteria were first hospital admission with SCI between 1 January 1989 and 1 May 2010, injury corresponding to the American Spinal Injury Association impairment scale (AIS) A-D at present investigation, SCI level at C4-L4 segment and $\geqslant 18$ years of age. Exclusion criteria were previous major gastrointestinal surgery and concomitant disease affecting gastrointestinal function. Because of the irradiation dose associated with this study, subjects with earlier irradiation towards the abdominal cavity or exposure to radiation $>5 \mathrm{mSv}$ above the background irradiation within the last year were not invited to participate.

\section{Bowel function scores}

Information about bowel symptoms and current medication in SCI subjects was collected using the international bowel function basic and extended SCI data set with a few additional questions. ${ }^{7,8}$ Based on this information, the NBD score, ${ }^{9}$ the St Mark's incontinence score ${ }^{10}$ and the Cleveland constipation score ${ }^{11}$ were calculated for each subject.

\section{Gastrointestinal transit time}

The method and data analysis are commonly used in other studies. ${ }^{4}$ Thereby, all SCI subjects took 10 radiopaque markers on 6 consecutive days. On day 7 ,

${ }^{1}$ Department of Surgery, Aarhus University Hospital, Aarhus, Denmark; ${ }^{2}$ Department of Neurosurgery, Aarhus University Hospital, Aarhus, Denmark; ${ }^{3}$ Neurogastroenterology Unit, Department of Hepatology and Gastroenterology, Aarhus University Hospital, Aarhus, Denmark; ${ }^{4}$ Department of Nuclear Medicine and PET Center, Aarhus University Hospital, Aarhus, Denmark and ${ }^{5}$ Department of Urology, Aarhus University Hospital, Aarhus, Denmark Correspondence: Dr MM Rasmussen, Department of Neurosurgery, Aarhus University Hospital, Noerrebrogade 44, Building no. 10, Aarhus, Aarhus C 8000, Denmark. E-mail: mikkrs@rm.dk

Received 19 February 2013; revised 17 April 2013; accepted 8 May 2013; published online 18 June 2013 


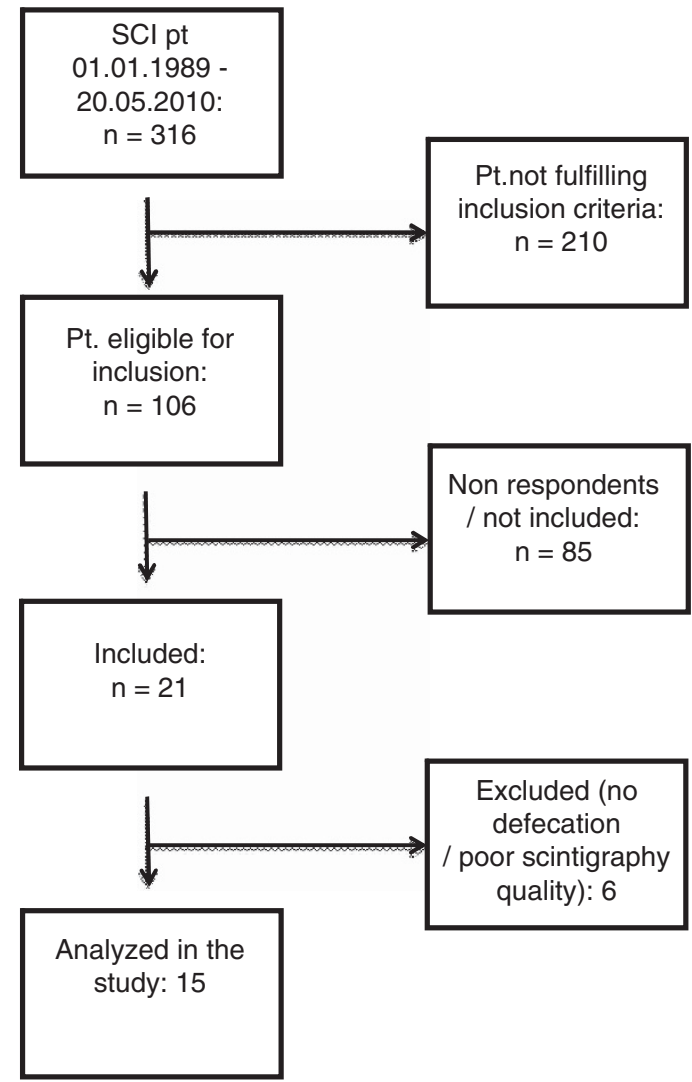

Figure 1 Flowchart of subject inclusion.

a whole abdominal X-ray was taken and GITT was calculated from the total number of markers present.

\section{Scintigraphic procedure}

Scintigraphic assessment of colorectal transport and bowel emptying during normal defecation has been described in detail by Krogh et al. ${ }^{12}$ Data from 16 of those healthy volunteers describing normal defecation in the previous study were used for comparison with SCI subjects in this study.

Subjects took ${ }^{111}$ In-coated resin pellets in $100 \mathrm{ml}$ of water for 2 days. To ensure marking of all colorectal content, subjects with GITT $\leqslant 2.5$ days took ${ }^{111}$ In-coated resin pellets 48 and $24 \mathrm{~h}$ before the study. If GITT $>2.5$ days, the ${ }^{111}$ In-coated resin pellets were taken 72 and $24 \mathrm{~h}$ before the study. All medication affecting gastrointestinal function, including Baclofen, was paused $24 \mathrm{~h}$ before the scintigram.

All investigations were performed at 0800 hours after an overnight fast and without subjects having defecated in the morning. Scintigraphy was performed before and after subjects had emptied their bowel using their normal bowelemptying procedures. Subject had a standardized breakfast just after the first scintigraphy and before bowel emptying to activate the gastrocolonic response.

Scintigraphy was performed using a double-headed Picker Axis gamma camera with parallel-hole, medium-energy collimators imaging the whole abdomen, with subjects lying flat on their back. The acquisition time was $10 \mathrm{~min}$ (five 2-min frames). Anterior and posterior images were obtained using $20 \%$ energy windows over the $174-\mathrm{keV}$ and $247-\mathrm{keV}{ }^{111} \mathrm{In}$ photopeaks, respectively.

\section{Computation of colorectal transport at defecation}

The number of counts in each segment was determined before and after bowel emptying. The colorectum was divided into four segments for analysis: the cecum and ascending colon including the right flexure, the transverse colon, the descending colon including the left flexure and the rectosigmoid. ${ }^{10}$ Correction for tissue attenuation was carried out by calculating the geometric mean (square root of each segment's anterior and posterior counts before and after defecation, respectively). ${ }^{13}$ Data were discarded if segments were overlapping in the scintigraphic frames making delineation of the segments impossible or if counts in the rectosigmoid were $>7.5 \%$ of the total counts prior to defecation.

Accordingly, defecation score and antegrade and retrograde segmental transport can be calculated as described in detail in an earlier publication. ${ }^{14}$ Briefly, a defecation score of 40 corresponds to emptying $40 \%$ of the content in the rectosigmoid during defecation and 150 corresponds to $100 \%$ emptying of the rectosigmoid and 50\% emptying of the descending colon. A maximum of 400 can be achieved, meaning all colonic content is lost at defecation. We calculated antegrade and retrograde transport from each segment. The transport is given in percentage of the content of the specific segment prior to defecation and is used in addition to the defecation score to describe movement within the colorectum at defecation.

\section{Statistical analysis}

Data analysis was performed using the statistical program STATA 12. Results were compared between healthy volunteers and SCI subjects using the Wilcoxon-Mann-Whitney test for statistical significance level. Associations between defecation score, symptom scores and GITT were tested with Spearman's rank correlation. Statistical significance was defined as $P<0.05$. Results are given as median and range.

\section{Ethical considerations}

The total whole-body effective radiation dosage was $1.1-3.1 \mathrm{mSv}$, depending on the individual GITT. The study was performed according to the Declaration of Helsinki II and approved by the local ethics committee for the Central Denmark Region (project no. M-20100299). We certify that all applicable institutional and governmental regulations concerning the ethical use of human volunteers were followed during the course of this study.

\section{RESULTS}

Out of 21 subjects with SCI, six were excluded due to overlapping colonic segments $(n=1)$, lack of defecation on the day of investigation $(n=1)$ and $<7.5 \%$ of total counts in the rectosigmoid segment prior to defecation $(n=4)$. Thus, 15 SCI subjects went on to further analysis (Figure 1). Patients were classified according to the International Standards for Classification of Spinal Cord Injuries. ${ }^{15}$ For comparison, 16 healthy volunteers were used. Table 1 describes detailed subject demographics.

\section{Colorectal emptying during defecation}

Examples of scintigrams from a subject with SCI and a healthy volunteer before and after defecation are shown in Figure 2. In SCI subjects, the median defecation score was 31 (range: 0-149), corresponding to $31 \%$ emptying of the rectosigmoid during defecation, and in healthy volunteers, it was 89 (range: 53-203), corresponding to $89 \%$ emptying of the rectosigmoid $(P<0.01)$. No association could be found to age $(P=0.10)$, AIS score $(P=0.82)$, time between SCI and investigation $(P=0.47)$ and SCI level $(P=0.94)$

\section{Segmental colorectal transport at defecation}

Comparing subjects with SCI and healthy volunteers, median antegrade transport was 5\% (range: $0-25 \%$ ) vs $4 \%$ (range: $0-39 \%$ ), $P=0.90$, from the ascending colon; $2 \%$ (range: $0-38 \%$ ) vs $6 \%$ (range: $0-130 \%$ ), $P=0.47$, from the transverse colon; $11 \%$ (range: $0-62 \%$ ) vs $30 \%$ (range: $0-73 \%$ ), $P=0.05$, from the descending colon and $31 \%$ (range: $0-194 \%$ ) vs $82 \%$ (range: 53-203\%), $P<0.01$, from the rectosigmoid. As illustrated in Figure 3, antegrade segmental transport was more affected by SCI in the distal than in the proximal part of the colorectum. 
Table 1 Display of subject demographics

\begin{tabular}{|c|c|c|c|c|c|c|}
\hline Group & $\begin{array}{l}\text { Age of investi- } \\
\text { gation (years) }\end{array}$ & Sex & $\begin{array}{c}\text { AIS score } \\
(A-D)\end{array}$ & $\begin{array}{l}\text { SCl level } \\
\text { (C5- Th9) }\end{array}$ & $\begin{array}{l}\text { Time since } \mathrm{SCl} \text { at } \\
\text { investigation (years) }\end{array}$ & Bowel-emptying procedure \\
\hline $\mathrm{SCl}$ & 22 & M & B & $\mathrm{C} 7$ & 4.6 & Clysma \\
\hline $\mathrm{SCl}$ & 32 & M & B & C6 & 4.3 & Digital stimulation, clysma \\
\hline $\mathrm{SCl}$ & 34 & M & B & $\mathrm{C5}$ & 1.7 & Clysma \\
\hline $\mathrm{SCl}$ & 41 & M & C & C6 & 1.5 & Digital stimulation, clysma \\
\hline $\mathrm{SCl}$ & 45 & M & A & $\mathrm{C7}$ & 1.8 & Digital evacuation, clysma \\
\hline $\mathrm{SCl}$ & 47 & M & A & Th8 & 2.2 & $\begin{array}{l}\text { Straning/bearing down to empty, } \\
\text { digital stimulation, digital } \\
\text { evacuation }\end{array}$ \\
\hline $\mathrm{SCl}$ & 55 & M & A & Th9 & 1.6 & Digital stimulation, clysma \\
\hline $\mathrm{SCl}$ & 63 & M & B & C5 & 2.3 & $\begin{array}{l}\text { Digital stimulation, digital eva- } \\
\text { cuation, clysma }\end{array}$ \\
\hline $\mathrm{SCl}$ & 65 & M & D & C5 & 2 & Digital stimulation, clysma \\
\hline $\mathrm{SCl}$ & $\begin{array}{c}74 \\
47(22-74)\end{array}$ & M & D & $\mathrm{C7}$ & $\begin{array}{c}4.5 \\
3.9(1.5-12.5)\end{array}$ & Clysma, digital stimulation \\
\hline Healthy volunteer & 24 & M & & & & \\
\hline Healthy volunteer & 24 & M & & & & \\
\hline Healthy volunteer & 24 & M & & & & \\
\hline Healthy volunteer & 27 & M & & & & \\
\hline Healthy volunteer & 27 & M & & & & \\
\hline Healthy volunteer & 36 & M & & & & \\
\hline Healthy volunteer & 39 & $\mathrm{~F}$ & & & & \\
\hline Healthy volunteer & $\begin{array}{c}42 \\
31(24-42)\end{array}$ & $\mathrm{F}$ & & & & \\
\hline
\end{tabular}

Abbreviations: AIS, American Spinal Injury Association impairment scale; F, female; M, male; SCI, spinal cord injury.

Median retrograde transport showed no difference between subjects with SCI and controls as it was $0 \%$ (range: $0-5 \%$ ) vs $0 \%$ (range: $0-36 \%$ ), $P=0.63$, from the transverse colon; $0 \%$ (range: $0-5 \%$ ) vs $0 \%$ (range: $0-25 \%), P=0.64$, from the descending colon and $0 \%$ (range: $0-21 \%$ ) vs $0 \%$ (range: $0-41 \%$ ), $P=0.57$, from the rectosigmoid.

\section{GITT and segmental colonic transit times}

Among SCI subjects, median GITT was 2.9 days (range: $1.5-5.8$ days), whereas transit time was 0.6 days (range: $0.1-3.4$ days) through the ascending colon, 0.2 days (range: $0.0-2.2$ days) through the transverse colon, 0.5 days (range: $0.0-1.7$ days) through the descending colon and 0.4 days (range: $0.0-1.7$ days) through the rectosigmoid. No correlation could be found between the scintigraphic defecation score and GITT $(P=0.99)$.

\section{Bowel symptoms}

In SCI subjects, the median NBD score was 10 (range: 3-23), corresponding to moderate bowel dysfunction. The median St Mark's fecal incontinence score was 4 (range: 0-10), and the median Cleveland constipation score was 6 (range: $4-13$ ). The scintigraphic defecation score was associated with the St Mark's fecal incontinence score $(P=0.02)$ but not with the NBD score $(P=0.12)$ and the Cleveland constipation score $(P=0.17)$. GITT was not associated with the St Mark's fecal incontinence score $(P=0.53)$, the NBD score $(P=0.83)$ or the Cleveland constipation score $(P=0.84)$.

\section{DISCUSSION}

We have demonstrated that subjects with supraconal SCI have significantly reduced emptying of the colorectum at defecation. Normal defecation usually includes emptying the majority of the 


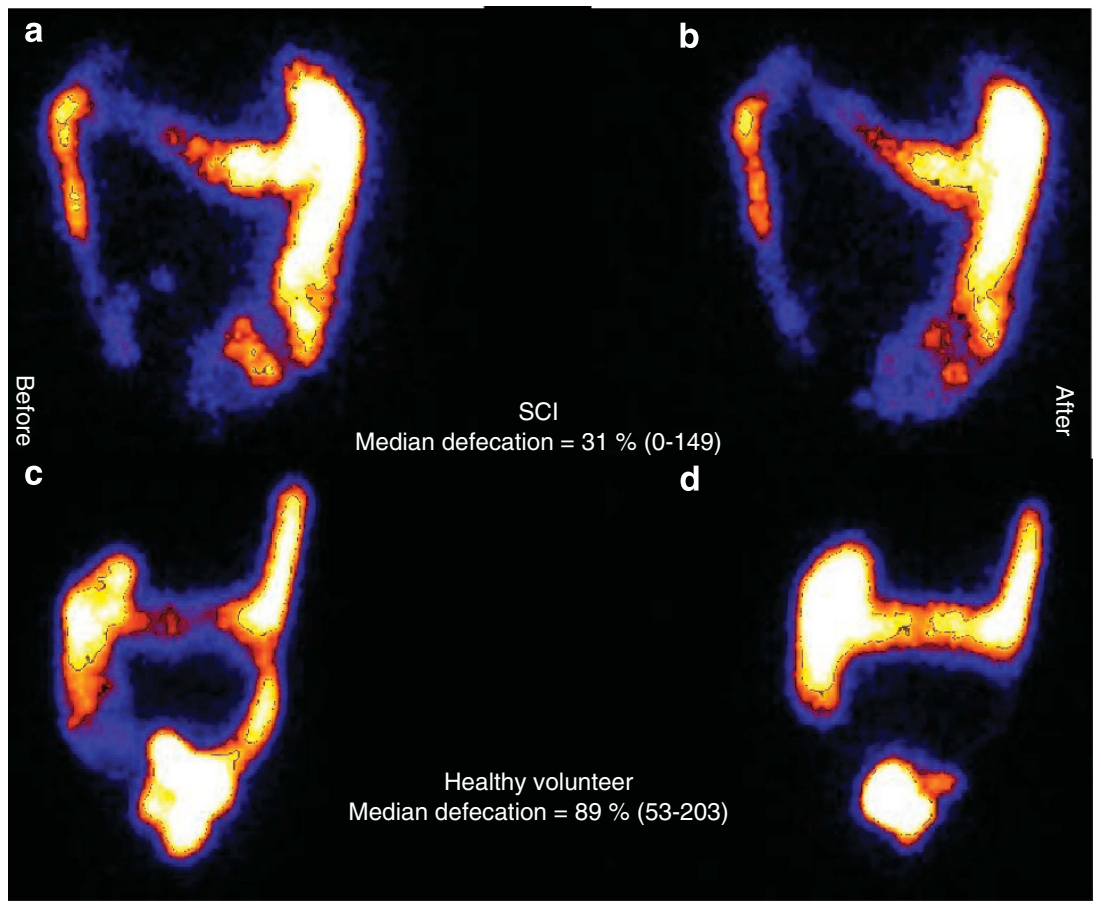

Figure 2 Colorectal scintigraphy before ( $\mathbf{a}$ and $\mathbf{c}$ ) and after ( $\mathbf{b}$ and $\mathbf{d}$ ) defecation for ( $\mathbf{a}$ and $\mathbf{b}$, at the top) an $\mathrm{SCl}$ subject and (c and $\mathbf{d}$, below) a healthy volunteer. As illustrated, a large proportion of the fecal matter in the descending colon and rectosigmoid is emptied during defecation by the healthy volunteer but not by the subject with $\mathrm{SCl}$.

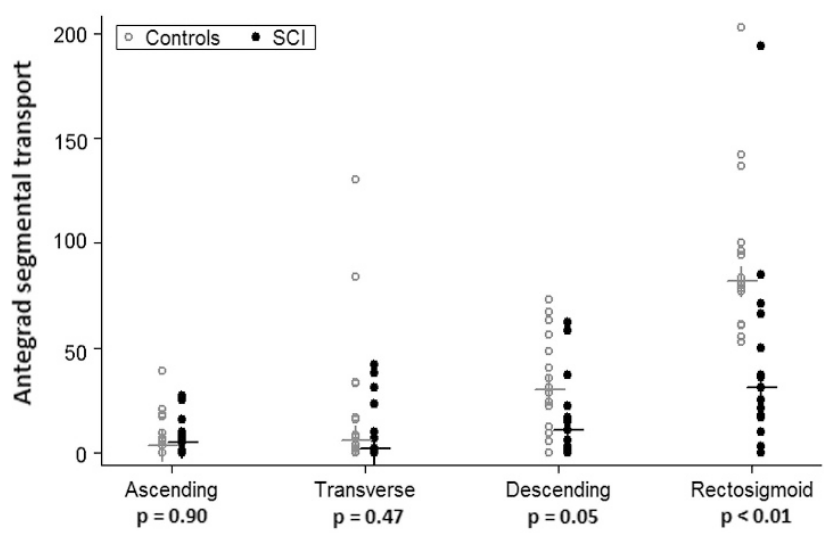

Figure 3 Display of the impaired bowel movement for the supraconal $\mathrm{SCl}$ subjects compared with healthy volunteers. Colorectal region of interest is displayed on the $x$ axis and percentage of predefecational content on the y axis. Impairment tends to increase as bowel content moves in anal direction. Primary impairment is seen in the left side of the colon (descending and rectosigmoid segments).

rectosigmoid and some antegrade transport from the transverse and descending colon. ${ }^{14}$ It is usually preceded by colonic mass movements that bring stools to the rectum. Distension of the rectal wall may further stimulate colorectal contractions by an intrinsic reflex mediated by the enteric nervous system and the parasympathetic defecation reflex involving spinal segments S2-S4. Rectal filling stretches the rectal wall and stimulates the rectoanal inhibitory reflex, relaxing the internal anal sphincter muscle. The rectoanal inhibitory reflex is intrinsic but probably enhanced by parasympathetic stimuli from S2 to S4. The defecation process is supported by a Valsalva maneuver.
Normal defecation physiology relies on intact neuromuscular control. Therefore, hampered evacuation and constipation-related symptoms are linked to SCI. ${ }^{1}$ There are, however, fundamental differences depending on the level of injury. Supraconal lesions result in increased colorectal tone and contractility, ${ }^{6,16}$ whereas conal and cauda equina lesions cause a hypotone and hyporeflex left colon and rectum. ${ }^{2}$ The rectoanal inhibitory reflex facilitating defecation is blunted in subjects with conal or cauda equina lesions but not in those with supraconal lesions. Accordingly, subjects with supraconal lesions in general have prolonged transit throughout the colon but less severely prolonged rectosigmoid passage. In contrast, subjects with conal or cauda equina lesions mainly have severely prolonged transit through the distal colorectum. ${ }^{2,12}$

These basic pathophysiological differences explain reduced emptying at defecation among subjects with conal/cauda equina lesions. ${ }^{12}$ The effect of supraconal SCI is less predictable. The hyperreactive rectal wall could ease digital stimulation and enhance the effect of suppositories or enemas, and bowel emptying is considered less difficult in supraconal than in conal/cauda equina lesions. ${ }^{17}$

In spite of this, data from this study clearly showed abnormally poor rectosigmoid emptying of stools in subjects with supraconal SCI. Further, antegrade segmental colorectal transport at defecation was significantly more pronounced in the distal than in the proximal parts. No difference was found in retrograde segmental transport. To what degree this is caused by reduced rectal compliance, ${ }^{18}$ rectoanal sphincter dyssynergia, ${ }^{19}$ blunted gastrocolonic response, loss of anorectal sensation or reduced abdominal muscle function remains to be investigated.

A study in a mixed population with multiple sclerosis and SCI has pointed towards an association between specific anorectal physiology markers and both the Wexner fecal incontinence score and Cleveland constipation scores, ${ }^{18}$ but anorectal physiology tests do not contribute 
much to the clinical evaluation of NBD. GITT is commonly used for evaluation of NBD in research and clinical practice. Unfortunately, there is no correlation between transit times and symptoms in subjects with SCI, ${ }^{5}$ and the present study could not find a correlation between the scintigraphic defecation score and GITT. This restricts the usefulness of GITT for clinical decision making.

It was our hope that colorectal scintigraphy would provide a valid alternative, also for clinical use. Even though data indicated a better correlation between symptoms and the scintigraphic defecation score than between symptoms and GITT, the only association reaching statistical significance was between the defecation score and the St Mark's incontinence score. The lack of statistical significance to the NBD score and the Cleveland constipation score may be a type II error caused by the inclusion of too few subjects. We believe that associations between symptoms and scintigraphic evaluation of defecation should be investigated in a larger study.

The association between poor emptying at defecation and fecal incontinence as observed in this study indicates that rectosigmoid impaction may contribute to fecal incontinence in supraconal SCI. The other factors are lack of voluntary control of the external anal sphincter muscle and loss of anorectal sensation. ${ }^{19}$ Perhaps a wider-scale use of irrigation should be considered for supraconal SCI subjects.

The colorectal scintigraphy method has been used for in several studies since its introduction ${ }^{20}$ and holds promise as an objective end point in future studies evaluating new and existing treatment modalities for NBD. It allows very detailed computations of defecation and colonic content movements. Limitations for the use of the method are availability, irradiation of subjects, insufficient pellet distribution in all colorectal segments and, with some protocols, the need for defecation at a specific time. To ensure representation of all colonic segments, two doses of pellets were taken and administered according to individual GITT. In spite of this, we had to exclude six subjects. The large variation in GITT (2.1-6.6 days in this study) is another limitation with the use of the method.

Of a total 106 eligible subjects, 21 were included and data from 15 were used. This reflects the reality of clinical research. It might have introduced selection bias, and the relatively small numbers may have caused type II errors where differences could not be found. However, the substantial difference between SCI and controls found in the study would not be affected by the small numbers. The gender distribution was not the same in SCI subjects and controls. Some earlier studies have found a difference in GITT between males and females. ${ }^{4}$ This was, however, not the case with colorectal scintigraphy. ${ }^{14}$ Age difference is another possible bias. Deterioration in continence with increasing age has been demonstrated but not in SCI subjects. ${ }^{6}$ In able-bodied controls, the subjective evaluation of whether defecation had been normal or not reflected the degree of colorectal emptying at defecation. ${ }^{14}$ Among subjects with SCI, we did not find the same correlation, probably due to reduced or absent anorectal sensation.

In conclusion, subjects with supraconal SCI have impaired colorectal emptying at defecation. Abnormally low emptying at defecation was associated with severe fecal incontinence, suggesting that rectosigmoid fecal impaction contributes to incontinence.

\section{DATA ARCHIVING}

There were no data to deposit.

\section{CONFLICT OF INTEREST}

The authors declare no conflict of interest.

\section{ACKNOWLEDGEMENTS}

We thank the Lundbeck Foundation for a grant supporting the completion of this study.

1 Glickman S, Kamm MA. Bowel dysfunction in spinal-cord-injury patients. Lancet 1996; 347: 1651-1653.

2 Krogh K, Mosdal C, Laurberg S. Gastrointestinal and segmental colonic transit times in patients with acute and chronic spinal cord lesions. Spinal Cord 2000; 38: 615-621.

3 Krogh K, Christensen P. Neurogenic colorectal and pelvic floor dysfunction. Best Pract Res Clin Gastroenterol 2009; 23: 531-543.

4 Abrahamsson H, Antov S, Bosaeus I. Gastrointestinal and colonic segmental transit time evaluated by a single abdominal X-ray in healthy subjects and constipated patients. Scand J Gastroenterol Suppl 1988; 152: 72-80.

5 Media S, Christensen P, Lauge I, Al-Hashimi M, Laurberg S, Krogh K. Reproducibility and validity of radiographically determined gastrointestinal and segmental colonic transit times in spinal cord-injured patients. Spinal Cord 2009; 47: 72-75.

6 Beuret-Blanquart F, Weber J, Gouverneur JP, Demangeon S, Denis P. Colonic transit time and anorectal manometric anomalies in 19 patients with complete transection of the spinal cord. J Auton Nerv Syst 1990; 30: 199-207.

7 Krogh K, Perkash I, Stiens SA, Biering-Sorensen F. International bowel function basic spinal cord injury data set. Spinal Cord 2009; 47: 230-234.

8 Krogh K, Perkash I, Stiens SA, Biering-Sorensen F. International bowel function extended spinal cord injury data set. Spinal Cord 2009; 47: 235-241.

9 Krogh K, Christensen P, Sabroe S, Laurberg S. Neurogenic bowel dysfunction score. Spinal Cord 2006; 44: 625-631.

10 Vaizey CJ, Carapeti E, Cahill JA, Kamm MA. Prospective comparison of faecal incontinence grading systems. Gut 1999; 44: 77-80.

11 Agachan F, Chen T, Pfeifer J, Reissman P, Wexner SD. A constipation scoring system to simplify evaluation and management of constipated patients. Dis Colon Rectum 1996; 39: 681-685

12 Krogh K, Olsen N, Christensen P, Madsen JL, Laurberg S. Colorectal transport during defecation in patients with lesions of the sacral spinal cord. Neurogastroenterol Motil 2003; 15: 25-31.

13 Hardy JG, Perkins AC. Validity of the geometric mean correction in the quantification of whole bowel transit. Nucl Med Commun 1985; 6: 217-224.

14 Krogh K, Olsen N, Christensen P, Madsen JL, Laurberg S. Colorectal transport in normal defaecation. Colorectal Dis 2003; 5: 185-192.

15 Maynard FM Jr, Bracken MB, Creasey G, Ditunno JF Jr, Donovan WH, Ducker TB et al. International Standards for Neurological and Functional Classification of Spinal Cord Injury. American Spinal Injury Association. Spinal Cord 1997; 35: 266-274.

16 Krogh K, Mosdal C, Gregersen H, Laurberg S. Rectal wall properties in patients with acute and chronic spinal cord lesions. Dis Colon Rectum 2002; 45: 641-649.

17 Yim SY, Yoon SH, Lee IY, Rah EW, Moon HW. A comparison of bowel care patterns in patients with spinal cord injury: upper motor neuron bowel vs lower motor neuron bowel. Spinal Cord 2001; 39: 204-207.

18 Thiruppathy K, Roy A, Preziosi G, Pannicker J, Emmanuel A. Morphological abnormalities of the recto-anal inhibitory reflex reflects symptom pattern in neurogenic bowel. Dig Dis Sci 2012; 57: 1908-1914.

19 MacDonagh R, Sun WM, Thomas DG, Smallwood R, Read NW. Anorectal function in patients with complete supraconal spinal cord lesions. Gut 1992; 33: 1532-1538.

20 Lubowski DZ, Meagher AP, Smart RC, Butler SP. Scintigraphic assessment of colonic function during defaecation. Int J Colorectal Dis 1995; 10: 91-93. 